BNL-112267-2016-JA

\title{
Sequential Programmable Self-Assembly: Role of Cooperative Interactions
}

\author{
Jonathan D. Halverson \\ Alexei V. Tkachenko' ${ }^{1,}$ Jonathan D. Halverson ${ }^{1}$ \\ ${ }^{1}$ Center for Functional Nanomaterials, Brookhaven National Laboratory, Upton, N.Y.
}

Submitted to the Journal of Chemical Physics

March 2016

Center for Functional Nanomaterials

Brookhaven National Laboratory

\section{U.S. Department of Energy Office of Basic Energy Sciences}

\footnotetext{
Notice: This manuscript has been authored by employees of Brookhaven Science Associates, LLC under Contract No. DE- SC0012704 with the U.S. Department of Energy. The publisher by accepting the manuscript for publication acknowledges that the United States Government retains a non-exclusive, paid-up, irrevocable, world-wide license to publish or reproduce the published form of this manuscript, or allow others to do so, for United States Government purposes.
} 


\section{DISCLAIMER}

This report was prepared as an account of work sponsored by an agency of the United States Government. Neither the United States Government nor any agency thereof, nor any of their employees, nor any of their contractors, subcontractors, or their employees, makes any warranty, express or implied, or assumes any legal liability or responsibility for the accuracy, completeness, or any third party's use or the results of such use of any information, apparatus, product, or process disclosed, or represents that its use would not infringe privately owned rights. Reference herein to any specific commercial product, process, or service by trade name, trademark, manufacturer, or otherwise, does not necessarily constitute or imply its endorsement, recommendation, or favoring by the United States Government or any agency thereof or its contractors or subcontractors. The views and opinions of authors expressed herein do not necessarily state or reflect those of the United States Government or any agency thereof. 
BNL-112267-2016-JA

\title{
Sequential Programmable Self-Assembly: Role of Cooperative Interactions
}

\author{
Jonathan D. Halverson ${ }^{1}$ and Alexei V. Tkachenko ${ }^{1}$ \\ Center for Functional Nanomaterials, Brookhaven National Laboratory, Upton, \\ New York 11973a)
}

We propose a general strategy of "sequential programmable self-assembly" that enables a bottom-up design of arbitrary multi-particle architectures on nano- and microscales. We show that a naive realization of this scheme, based on the pairwise additive interactions between particles, has fundamental limitations that lead to a relatively high error rate. This can be overcome by using cooperative interparticle binding. The cooperativity is a well known feature of many biochemical processes, responsible e.g. for signaling and regulations in living systems. Here we propose to utilize a similar strategy for high precision self-assembly, and show that DNAmediated interactions provide a convenient platform for its implementation. In particular, we outline a specific design of a DNA-based complex which we call "DNA spider", that acts as a smart interparticle linker and provides a built-in cooperativity of binding. We demonstrate versatility of the sequential self-assembly based on spider-functionalized particles by designing several mesostructures of increasing complexity and simulating their assembly process. This includes a number of finite and repeating structures, in particular, the so-called tetrahelix and its several derivatives. Due to its generality, this approach allows one to design and succesfully self-assemble virtually any structure made of a "GEOMAG" magnetic construction toy, out of nanoparticles. According to our results, once the binding cooperativity is strong enough, the sequential self-assembly becomes essentially error-free.

PACS numbers: 81.16.Dn,87.14.gk,81.07.-b

a)Electronic mail: Electronic address: oleksiyt@bnl.gov 


\section{INTRODUCTION}

The field of colloidal and nanoparticle self-assembly has been dramatically transformed in the last decade due to an introduction of smart DNA-mediated interactions ${ }^{1-9}$. While most of the progress has been made in self-assembly of various periodic superlattices, a natural next frontier for this field would be the programmable mesoscopic architecture. Specifically, one could use the molecular recognition properties of DNA to design interparticle interactions in such a way that they self-assemble into multiparticle constructs with a prescribed overall shape and local arrangement. This challenge has been attacked theoretically and numerically in a number of recent works ${ }^{10-15}$..

In its core, the problem is two-fold. First, one has to ensure that a target structure is indeed a ground state of the system of interacting building blocks. Second, there must be a fast kinetic pathway leading towards that ground state, preferably without unwanted metastable configurations. Ideally, this targeted self assembly should happen in a solution of dispersed building blocks, without a need of isolation of a precise set of constituent particles. In our previous works on this subject Ref. ${ }^{16}$, we have demonstrated that the use of nanoparticles (NPs) with directional DNA-coded interactions allows to solve the underlying "inverse self assembly problem". We have shown that a set of such particles can be designed in such a way that the target multi-particle cluster is both the equilibrium ground state and the kinetically preferred configuration.

In this paper we propose a concept of "sequential programmable self assembly" which is applicable beyond that specific class of directionally-functionalized particles. The central idea of this approach is illustrated in Figure 1. Consider a set of spherical particles that have to self-assemble into clusters with a specific target architecture. We propose to achieve this by designing interparticle interactions in such a way that each particle typically binds to a partially built cluster only after $D$ of its nearest neighbors are already in place, where $D$ is space dimensionality. By forming these $D$ contacts, the particle will automatically be placed in a correct position.

In this paper, we analyze two distinct mechanisms that can be used for achieving this sequential assembly regime. The simpler one, that we call "coordinated binding" relies on pairwise-additive interparticle interactions. If the strengths of these interactions can be tuned, as in the case of DNA-functionalized particles, one can achieve conditions in which 


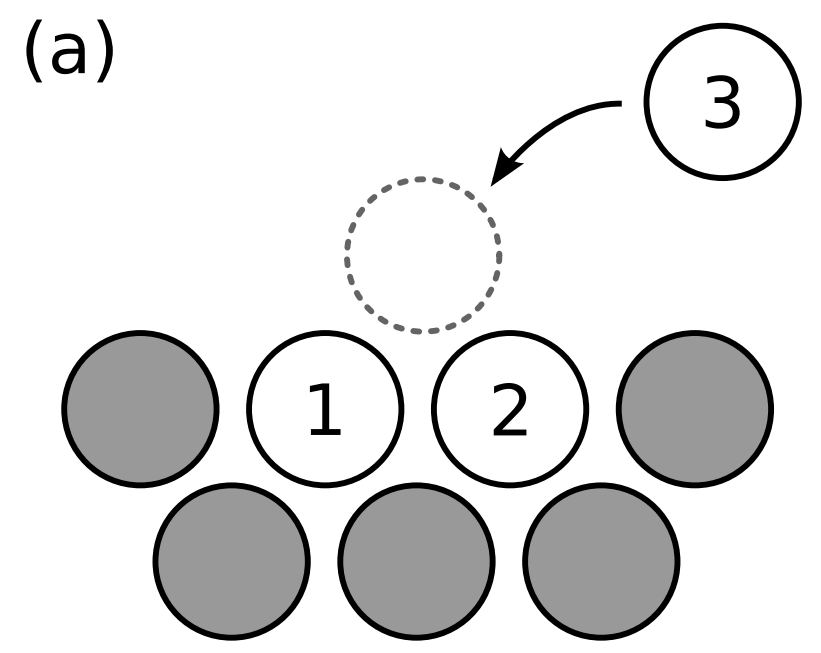

(b)

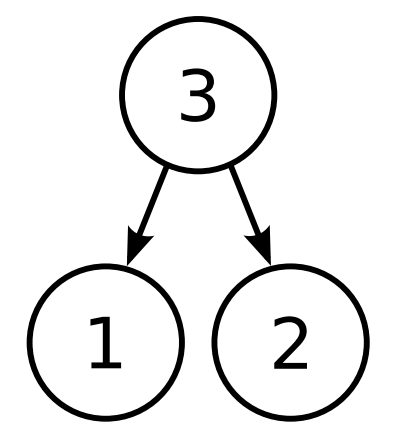

FIG. 1. The concept of sequential programmable self-assembly. (a) Target cluster with the last particle in the process of binding. (b) Graph showing the interaction between the three particle types involved.

a single bond is not strong enough to keep the particles bound, but having $D$ bonds formed is sufficient to hold the newly arrived particle in place, as shown in Fig. 1. As we will demonstrate, the "coordinated binding" strategy has fundamental limitations that make it not practical. We will therefore focus primarily on a more sophisticate version of the sequential assembly that relies on "cooperative binding". Here we use term "cooperative" in its classical biochemical sense, i.e. we study the system in which existing bonds change the affinity of the consecutive ones. A classical example of cooperative binding is the interaction of Hemoglobin with oxygen. Hemoglobin is an iron-containing protein responsible for carrying oxygen from the lungs and transporting it through the blood to the body. Mammalian hemoglobin is capable on binding to four oxygen molecules. It has been shown that the binding affinity between hemoglobin and oxygen increases with the addition of each oxygen molecule. This is known as positive cooperative binding and it explains many biochemical and physiological processes. As we will demonstrate, the DNA-based interactions open an opportunity of designing particles with such cooperative binding properties, and this cooperativity is key for a successful implementation of the sequential programmable self-assembly. 


\section{COORDINATED AND COOPERATIVE BINDING}

We first consider the simplest realization of the sequential self-assembly scheme that relies on the "coordinated" binding, i.e. the one in which interactions between particles are pairwise additive. Its Hamiltonian can be written as:

$$
\frac{H}{k_{B} T}=\sum_{\left|\vec{r}_{i}-\vec{r}_{j}\right|=R_{i}+R_{j}} \varepsilon_{i j}
$$

Here the particles behave as sticky spheres with type-dependent affinity. The interaction matrix $\varepsilon_{i j}$ is chosen in such a way that a specific target structure is favored:

$$
\varepsilon_{i j}=\left\{\begin{array}{l}
\epsilon, \text { if particles 'i' and ' } \mathrm{j} \text { ' to be neighbors } \\
0, \text { otherwise }
\end{array}\right.
$$

Strictly speaking, the condition $\left|\vec{r}_{i}-\vec{r}_{j}\right|=R_{i}+R_{j}$ should be replaced with an interaction potential with certain localization length $\delta$. We assume this length is much smaller than the particle size and adsorb the entropic effect of the localization within the binding free energy: $\epsilon=\epsilon_{0}-\ln \left(\delta / V^{1 / 3}\right)(\epsilon<0)$. Here $\epsilon_{0}$ is the binding energy, and $V$ is a single particle volume ( $V^{1 / 3}$ is used as a reference length scale in our model). With this definition of $\epsilon$ we can "drop" one degree of freedom for each newly formed bond without loss of consistency in calculating the overall partition function of the system.

The collective effect arises because a newly added particle with $D$ favorable contacts, has lower free energy than the one with a smaller number of bonds. Automatically, this number

of contacts enforces the prescribed position of such a particle with respect to the rest of the cluster. There are two sources of errors that one can anticipate in this type of sequential self-assembly process: (i) binding of "dangling" particles with insufficient number of bonds and (ii) occurrence of a vacancies in place of expected particles.

The probability of attaching a "dangling" particle that has $D-1$ bonds can be expressed as

$$
p_{+}=A \phi\left[\frac{\delta}{V^{1 / 3}}\right]^{D-1} \exp \left[-(D-1) \epsilon_{0}\right]=A \phi \exp [-(D-1) \epsilon] .
$$

Here $\phi$ is the volume fraction of particles of a given type that is expected to form $D$ binds with the current cluster, but in this case only forms $D-1$ of them. Integration over one degree of freedom that remains unconstrained results in a dimensionless prefactor 
$A \sim 1$. According to this expression, in order to avoid "dangling" particles, one should decrease the bond strength $|\epsilon|$. This, however, would result in an increased probability of a vacancy. The latter can be estimated in a two-state approximation, i.e. by considering only "occupied" and "unoccupied" states whose statistical weights are given by 1 and $\phi \exp (-z \epsilon)$, respectively:

$$
p_{-}=\frac{1}{1+\phi \exp (-D \epsilon)} \approx \frac{\exp (D \epsilon)}{\phi} .
$$

He we assume that the probability of a vacancy is small compared to the "occupied" state.

As one can see, the two types of errors (dangling particles and vacancies) cannot be suppressed simultaneously by changing the binding free energy $\epsilon$. In fact, one can construct an invariant of these two error probabilities that is completely independent of $\epsilon$ :

$$
p_{-}^{D-1} p_{+}^{D}=A^{D} \phi \sim \phi
$$

This result imposes a serious limitation on the sequential programmable self-assembly scheme based on pairwise-additive interactions. Namely, in order to achieve a sufficient error suppression, one needs to work at extremely low particle densities. Specifically, if we require both types of error to be about 1\%, the volume fraction of particles of each type must be below $0.01^{2 D-1}$, i.e. $10^{-6}$ in $2 \mathrm{D}$ and $10^{-10}$ in $3 \mathrm{D}$. This constraint makes its experimental implementation nearly impossible, especially in the 3D case. One can probably achieve a satisfactory yield by using non-spherical particle, but that scenario has its own limitations. Instead, below we focus on sequential programmable self-assembly that relies on cooperative binding.

As we have discussed earlier, cooperativity plays a prominent role in many biochemical regulatory processes. Here we propose to use the same mechanism in the context of selfassembly. The key idea is to design the interactions in such a way that the arrival of one particle increases the probability of consecutive bindings, by changing the corresponding interparticle affinities. The simplest way in which it can be introduced to our model is by assigning an additional "bonus" free energy $-k_{B} T \Delta$ to particles that have coordination number $Z$ larger or equal to the number of spacial dimensions, $D$. This bonus significantly changes the above calculations of the error probabilities. In particular, the probability of a 
vacancy decreases exponentially:

$$
p_{-} \approx \frac{\exp (D \epsilon+\Delta)}{\phi}
$$

As a result, the constraint on the volume fraction becomes far less severe:

$$
p_{-}^{D-1} p_{+}^{D} \sim \phi \exp (\Delta)
$$

\section{COOPERATIVITY BY DESIGN: "DNA-SPIDER"}

Due to the versatility and tunability of DNA-based interactions, one can use them to design particle systems with a built-in cooperativity. In particular, Fig. 2 illustrates one of the possible approaches to achieve this. It is based on the use of multi-DNA constructs that we will refer as "DNA spiders". In the case of 3D self-assembly it consists of 3 DNA chains that have pairwise complementary segments resulting in a star-like binding configuration. The tails of this three-DNA complex are "sticky" allowing them to irreversibly bind to a particle surface, e.g. by using thiols or other chemical groups. As a result, one obtains a new type of "designer particles" that have one or more 3-DNA "spider" sites. An advantage of the spider complex is that the DNA strands are originally bound together, and have certain negative free energy associated with this. In order to use these DNA strands to bind other DNA-functionalized particles, their original star-like structure needs to be destabilized. However, once two external bonds are formed, the penalty for breaking the original spider ground state is already paid, and the third bond becomes more probable. This gives rise to the cooperativity and ultimately leads to a drastic suppression of errors in sequential self-assembly.

More quantitatively, let us assume that the spider is formed by three DNA strands, 'a', 'b', and 'c' whose mutual interaction is characterized by the ground state free energy $\Delta G_{a b c}<0$ , and by two-strand binding free energy, $\Delta G_{a b}, \Delta G_{b c}$ and $\Delta G_{a c}$. Each of the DNA strands

in the spider can be connected to the corresponding DNA-functionalized particle of type A, $\mathrm{B}$, or $\mathrm{C}$, and the external binding free energy is given by $\Delta G_{a}, \Delta G_{b}$ and $\Delta G_{c}$, respectively. Consider a situation when the spider has already formed two external bonds, e.g. strands 'a' and 'b' are bound to the complementary ssDNA belonging to particles A and B, respectively. The overall binding free energy can be written as 
I.

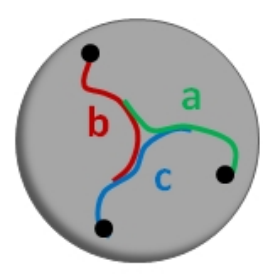

III.

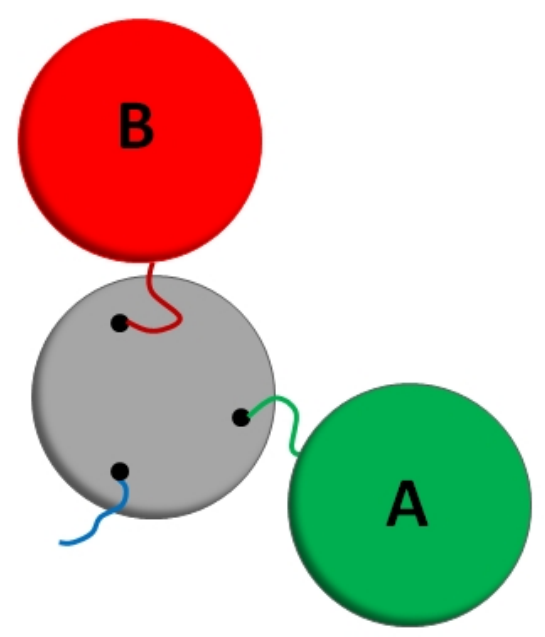

II.
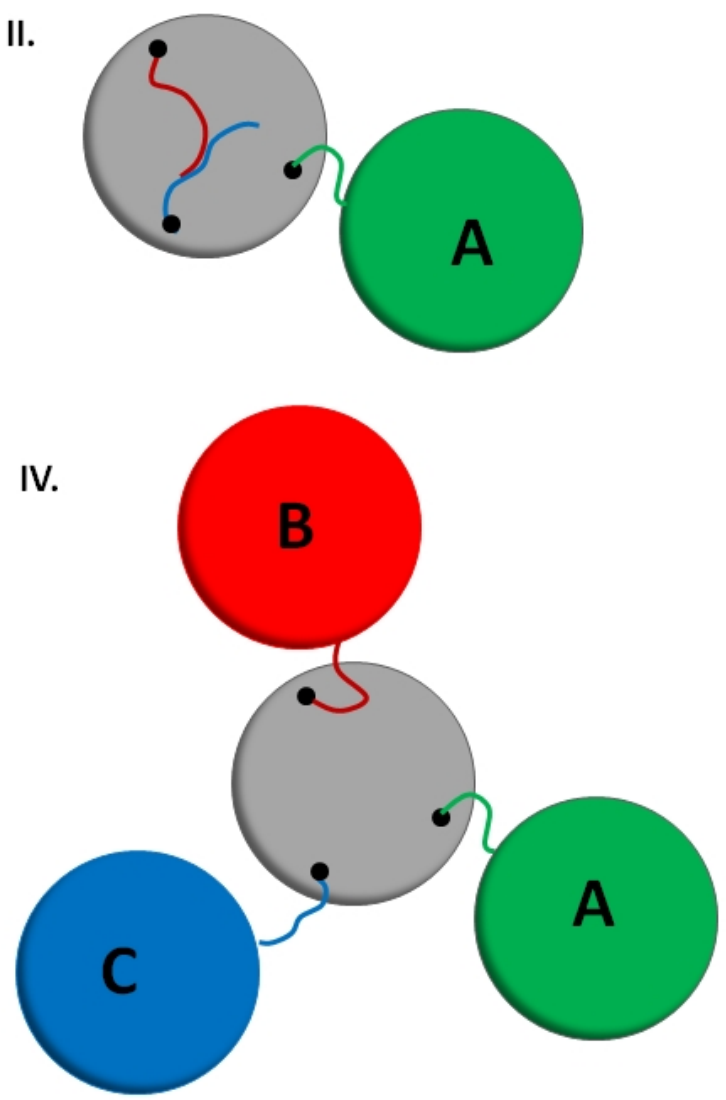

FIG. 2. Illustration of the proposed DNA-"spider" construct and process of binding of "spider"functionalized particle to its target $A B C$ triplet. (I) represent unbound particle, with DNA "spider" in its star-like ground state; (II)-(IV) show consecutive binding to 1, 2 and 3 target particles of types $A, B$ and $C$, respectively.

$$
\Delta \tilde{G}_{a}+\Delta \tilde{G}_{b}=\Delta G_{a}+\Delta G_{b}-\Delta G_{a b c}
$$

Here we introduced an effective binding free energy $\Delta \tilde{G}_{i} \equiv \Delta G_{i}-\Delta G_{a b c} / 2$. Upon this redefinition, the free energy of binding to all three external particles (A, B and C) can be expressed as:

$$
-\Delta G_{a b c}+\sum_{i=a, b, c} \Delta G_{i}=\frac{\Delta G_{a b c}}{2}+\sum_{i=a, b, c} \Delta \tilde{G}_{i}
$$

One can see that the three-body binding is indeed cooperative with $\Delta=-\frac{\Delta G_{a b c}}{2 k_{B} T}$. Furthermore, if we consider an effective strength of a single external binding, e.g. to particle A 
only, it turns out to be suppressed due to a positive free energy penalty $\Delta G_{b c}-\Delta G_{a b c} / 2$ :

$$
\Delta G_{a}+\Delta G_{b c}-\Delta G_{a b c}=\Delta \tilde{G}_{a}+\left[\Delta G_{b c}-\frac{\Delta G_{a b c}}{2}\right]
$$

This is another manifestation of the cooperativity in the system. However, for simplicity we will neglect this effect in the computational model presented below because single-bond particles are rather rare even without this correction. Additional advantage provided by the spider design is that it provides time-like ordering between interacting particles. Specifically, when a spider-functionalized particle finds its target position, the triplet of particles to which it binds can be thought of being "upstream", while the new particle is "downstream" from the point of view of the assembly instruction. Note that the mutual interactions between the DNA strands that make up a single spider can be related to other schemes, both theoretical and experimental, in which interactions between DNA strands that belong to the same particle lead to rather non-trivial overall behavior ${ }^{6,17}$.

It should be noted that the experimental implementation of DNA spider system will most definitely require resolving a number of technical and design issues. For instance, the typical size of the spider and linkers should be comparable to the particle radius. This makes this scheme more applicable to nanoparticles than to micron-size colloids. However, most of the results reported below are expected to be generic for any system that features cooperative binding, and therefore major conclusions should be applicable beyond the specific case of DNA spiders.

\section{SIMULATION PROCEDURE}

In our computational model, each particle is isotropically functionalized with one type of implicit ssDNA. In the figures below, particles of the same color have the same isotropic functionalization. Additionally, particles can have DNA "spider" sites which determine the set of isotropic particles to which they can bind. In our model, spider site is a point on the surface of a particle, that can be "connected" to other particles with a harmonic spring, in a stochastic manner.

We engineer a number of increasingly complex mesostructures, and use Brownian dynamics simulations to verify that the mesostructures successfully self-assemble from an initially disordered solution of particles. In the simulations, particles undergo translational and rota- 


\section{additive binding}

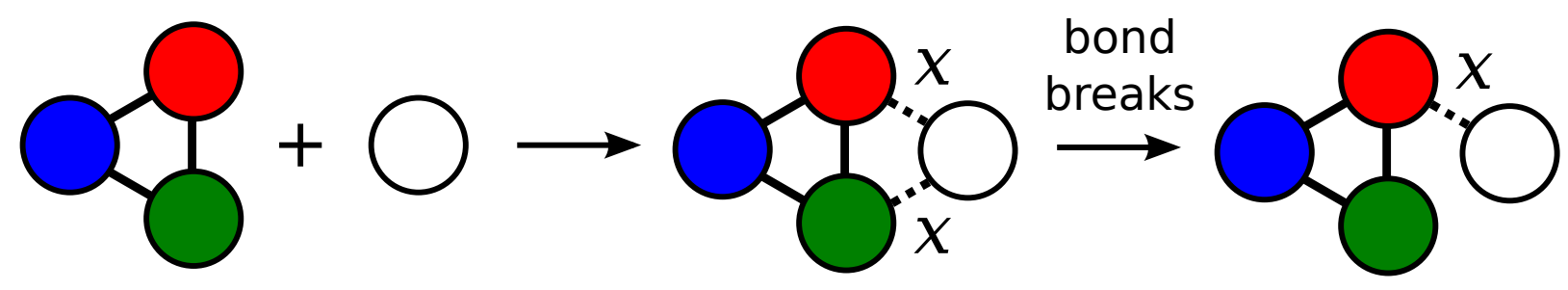

\section{"spider" binding}
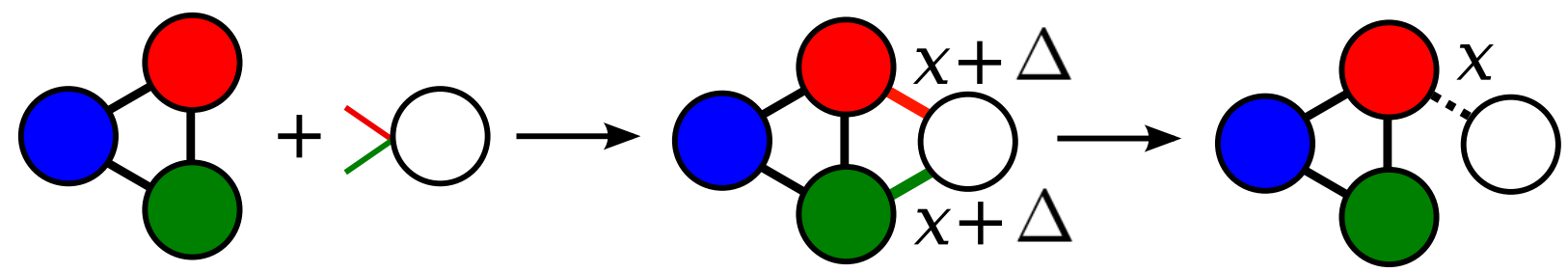

FIG. 3. Illustration of the difference between additive ("coordinated") binding and cooperative "spider"-based binding and its implementation in our numerical model. With additive binding the free energy decreases in proportion to the number of bonds formed. With the spider sites model, the binding energy is non-additive. The $x$ parameter is a measure of the deviation from the melting temperature of a single bond. However once $D$ bonds are formed ( $D=2$ in this illustration), the system gains bonus free energy $-k_{B} T \Delta$. This means that breaking any of the two bonds becomes exponentially harder. Once the bond is broken, the strength of the remaining one returns to the original value $x$.

tional Brownian motion subject to an excluded-volume potential $U_{\text {ex }}(r)=A \exp [-\kappa(r-2 a)]$, ( $r$ is the separation distance and $a$ is the particle radius), as well as to a harmonic DNA bonds $U_{\text {bond }}(l)=k_{s}\left(l-l_{0}\right)^{2} / 2$. The bond forms between the center of the isotropic particle and the spider site, with $l$ being the distance between them, $l_{0}=a / 2$, and $k_{s}$ playing the role of effective spring constant.

The particle volume fraction is $\phi=(4 / 3) \pi a^{3} N / L^{3} \sim 10^{-3}$, where $N$ in the number of particles and $L$ is the side of the cubic simulation box. Periodic boundary conditions were used.

The forming and breaking of dsDNA bonds was treated using a stochastic scheme based on a local equilibrium model. For each bond type, the dissociation and association rate constants are related as 


$$
k_{d}=k_{a} \frac{C}{4} e^{x_{i}},
$$

Here parameter $x_{i}$ is closely related to the effective hybridization free energy of the corresponding DNA bond: $x_{i} \equiv \Delta \tilde{G}_{i} / k_{B} T+\log \left(C / C_{0}\right) . C=N / L^{3}$ is the concentration of the particles with a given spider type, and $C_{0}$ is a standard reference concentration, typically $1 \mathrm{M}$. One can see that the particle concentration does not really affect the local kinetics of bond formation and breaking since $C$ under $\log$ in the definition of $x_{i}$ cancels with $C$ in the denominator of the right hand side of the above equation. Nevertheless, it is convenient to adsorb concentration within the definition of $x$. In this way $x=0$ has a very clear physical meaning of the melting point of a given DNA duplex (corrected by the need of breaking the internal DNA bond within a spider). As mentioned above, we neglect the cooperative correction that leads to an additional free energy penalty for single-bond particles. In other words, the binding free energy for a spider remains additive as long as the number of bonds is less than $D$. However, once the $D$-th bond is formed, the system gains additional bonus free energy $-k_{B} T \Delta$. The overall numerical procedure, aside from treatment of cooperativity, is essentially identical to the one described in our previous work, Ref. ${ }^{16}$. The further simulation details can be found in its Supplementary Information section.

Our modeling of the cooperative interactions is illustrated in Fig. 3. In the figure, the white particle has a "spider site" on its surface. In the model, the lines serve to indicate the types of particle which may bind. The types are indicated by the color of the lines which is red and green in this case. In general, when $D-1$ bonds exist between the spider particle and its downstream bonding partners, all bonds are of strength $x$. However, when the $D$ bonds are formed the $x$ value becomes $x+\Delta$ for all $D$ bonds. This causes the bonds to be very strong in the case of large $\Delta$. The strength of the bonds is checked at every time step. If one or more of the $D$ bonds breaks then all of the remaining bonds return to their previous strength of $x$. Note that through this procedure when fewer than $D$ particles are present the enhanced bonds are not possible. This leads to suppressed spontaneous nucleation: when only a few particles collide, they can form weak bonds not enforced by a cooperative free energy bonus, and therefore these bonds typically have a short lifetime. However, when the particle binds to one of its $D$ prescribed upstream neighbors in the cluster it will most likely bind with the others too, resulting in a strong strong cooperative bond (given that the full set of $D$ upstream particles is available). Note that $\Delta=0$ corresponds to simple additive 
interactions with no cooperativity.

We imagine the seed being pre-assembled from distinct DNA-functionalized NPs, possibly with the use of DNA origami or other type of DNA nanotechnology ${ }^{18}$. In the simulations the bonds between the particles in the seed are permanent. The seed NPs do not have any spider sites. In this work we consider both tetrahedron and icosahedron seeds but many other choices exist. In fact, below we demonstrate that as a cluster grows it can create new sites which lead to branching or the growth of new structures seeded from sites in the original cluster. In general, the role of the seed is to serve as an original nucleation site to initiate assembly. In this way, the seeds also control the total number of number of clusters being assembled. Other constituent particles are typically present in excess.

\section{NUMERICAL RESULTS}

\section{A. Non-cooperative sequential assembly}

The argument above suggests that it is unfeasible to assemble NP clusters based on coordinated (non-cooperative) binding in 3D. However, in 2D the required concentration is more practical. First, we have conducted simulations in $2 \mathrm{D}$ with the cooperativity parameter $\Delta$ set to zero, to test this prediction.

The seed is a triangle composed of particles $B, C$ and $D$ (see inset of Fig. 4). Particles $B$ and $C$ are capable of forming bonds to the "spider" of the free $A$ particles (see Fig. 4). As the volume fraction is decreased, we observe that the errors decrease and the yield of the target approaches relatively high values. As was predicted, in this $2 \mathrm{D}$ case the concentration needed for a reasonable yield is in practical range: the yield is approaching $70 \%$ for area fraction of A particles around $10^{-3}$. Figure 5 shows a yield and error probability in a similar non-cooperative assembly in $3 \mathrm{D}$, in which case a single particle is suppose to bind to a face of a tetrahedral seed. As expected, the yield remains modest and shows a very slow improvement with a decreasing volume fraction, if any at all (the sudden drop in yield at $\phi=10^{-5}$ is probably an artifact of insufficient statistics). Our simulations generally support the prediction that the sequential self-assembly is prone to errors in the non-cooperative (additive) binding regime. 


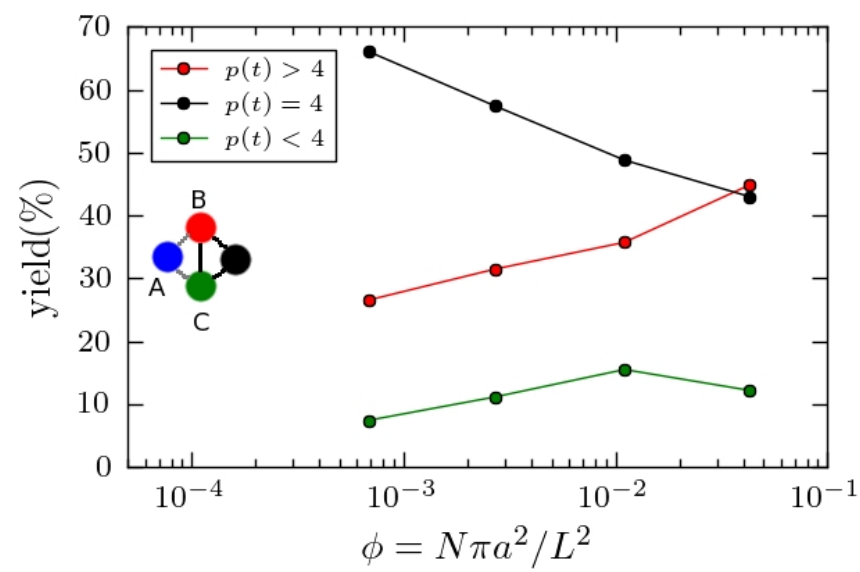

FIG. 4. Isotropic interactions: Yield of the target and error probabilities as a function of $\phi$ for a 2-dimensional system with $N=39$. Particles B and C encode for the binding of A. $p(t)$ represent a time average probability of finding a cluster of a given size, 4 being a target

\section{B. Finite and periodic designs}

The first cooperative system that we consider is the 7-particle cluster in 3D obtained by a targeted addition of three particles to a tetrahedron seed. The first particle binds to three particles that make one of the faces of the seed. A second particle then binds to two of the seed particles and the previous new particle. Lastly, the third particle binds to one of the seed particles and the two new particles. We have conducted a series of simulations of this system for a wide range of the cooperativity parameter $\Delta$. The yield of the 7 -particle cluster as a function of $\Delta$ is shown in Fig. 7 . As the $\Delta$ parameter is increased the yield of the target approaches $100 \%$ while that of the unwanted structures goes to zero. As $\Delta$ increases the $x$ value can also be increased which causes unwanted particles to break away immediately. One should keep in mind that the remaining free particles are occasionally binding to the target but break off very quickly.

This result establishes the feasibility of sequential self-assembly aided by cooperative in- 


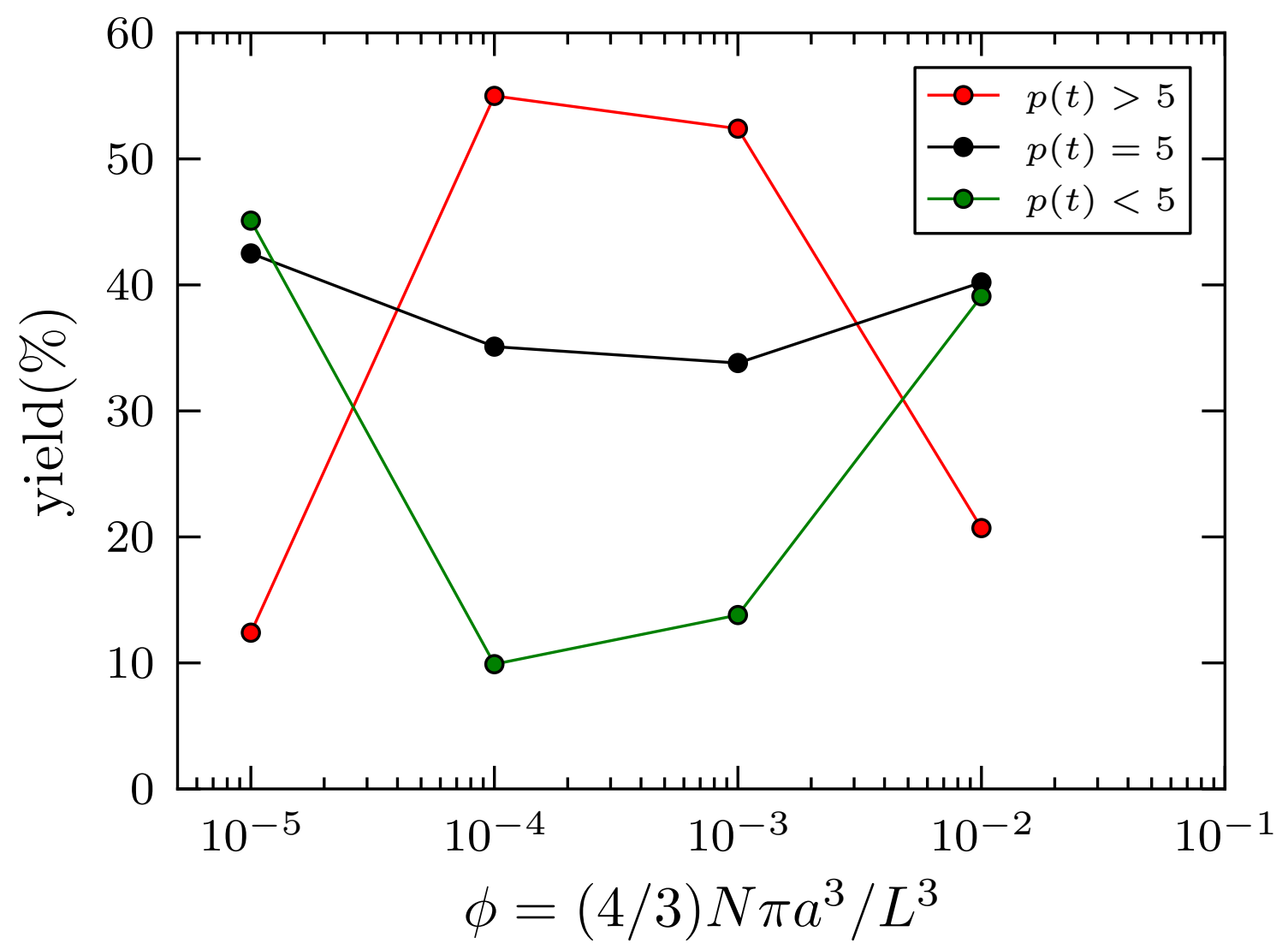

FIG. 5. Yield of the target as a function of volume fraction in 3D. Tetrahedron seed plus 64 particles of the same type $(\Delta=0)$.

teractions, a strategy that can be used to obtain a great variety of programmed architectures . The only essential requirement is that each upstream particle should have $D=3$ downstream neighbors. The obvious challenge in this approach is the need to use a large pool of distinct specially designed spider-functionalized particles. In the most general case, each particle in the target cluster should be encoded by a unique particle type with a specific spider. Fortunately, as we have previously demonstrated for the case of directionally functionalized NPs ${ }^{16}$, one can greatly reduce the number of particle types if the target structure possesses certain symmetries.

Specifically, one can go beyond finite clusters by designing a set of particles that generate a repeat unit. This is achieved by having the particles regenerate the initial face of the seed which initiated the growth. The design that we have studied first is a natural extension of the above finite cluster: a spider of type- $n$ particle links to the downstream particles of type $n-1, n-2$, and $n-3$. In this homogeneous design, one can limit the number of particle types to number $\mathrm{Z}$ by making types of particles $n$ and $n+Z$ mutually equivalent. This interaction rule leads to a famous structure known as the BoerdijkCoxeter helix ${ }^{19,20}$, 


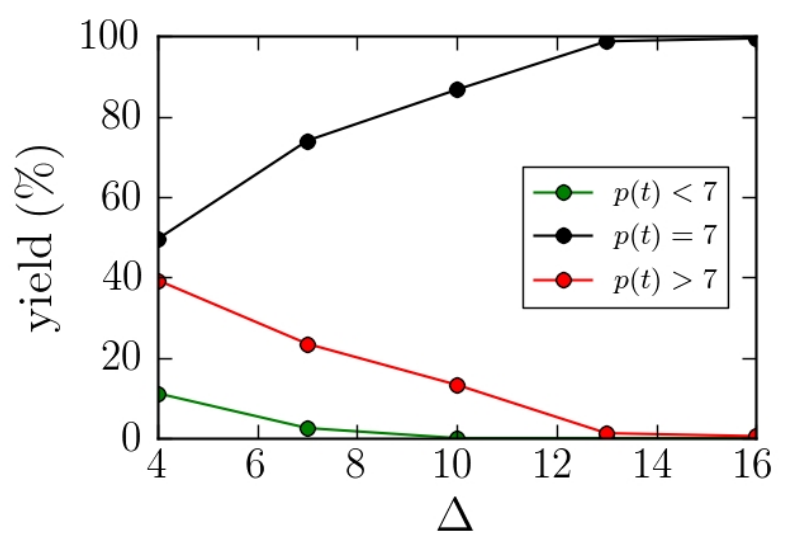

FIG. 7. Yield versus $\Delta$ for a tetrahedron seed with three particles designed to add to one face of the seed.

has 6 neighbors (3 upstream, and 3 downstream), and all of them should be distinct to avoid erroneous binding.

\section{Increasing design complexity}

Now, we can easily modify the design of the tetrahelix to achieve alternative morphologies. For instance, Fig. 9 represents an interaction graph that closely resembles that of a classical tetrahelix, but underwent a modest "rewiring": the spiders that correspond to particles '1' and '2' are switched, and that of particle '3' binds to triplet ('6', '1', '2') instead of ('7','1', '2'). That change is sufficient to produce strikingly different helical structure shown in Fig. 8 (c), which we call "modified tetrahelix". Unlike the original one, this structure has a pronounced helical shape with both radius and pitch close to $10 \mathrm{a}$. Again, the assembly is 
(b) tetrahelix

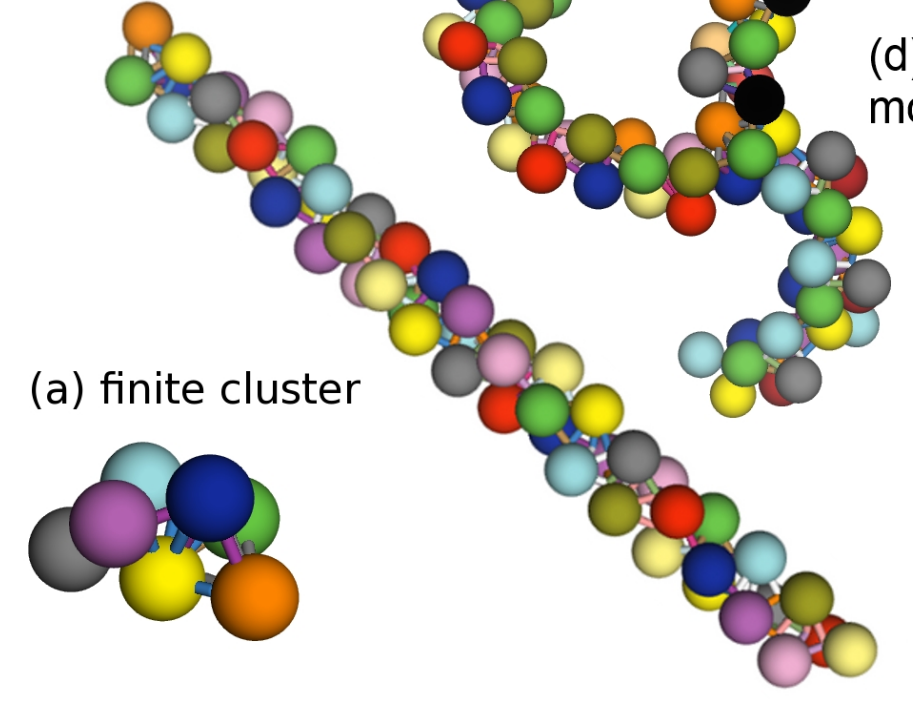

(d) three-armed modified tetrahelix

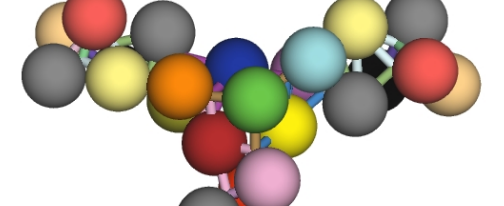

finit

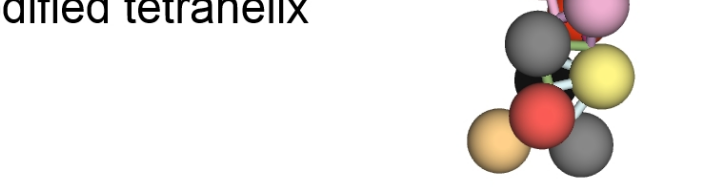

FIG. 8. Examples of structures that have been successfully designed and self-assembled in our simulations: (a) 7-particle finite cluster, (b) tetrahelix with $Z=10$ particle types; (c) modified tetrahelix, $Z=7$, with interaction rules defined by the connectivity graph in Fig. 9; (d) threearmed finite cluster; (d) three-armed modified tetrahelix; and (e) modified tetrahelix initiated at an icosahedral seed.

essentially error-free.

Note that the chirality of both modified and non-modified tetrahelices is not spontaneous but pre-programmed, despite the fact that interactions themselves are not chiral. Instead, it is completely predetermined by the original "coloring" of the seed. Furthermore, one can selectively initiate assembly at specific faces of the seed. For instance, this can lead to propeller-like multi-arm assemblies, both finite ones and those with unrestricted growth, shown in Fig 8 (d)-(e). Use of alternative seeds, such as icosahedron further diversify the range of plausible morphologies (see Fig. 8 (f)).

As the mesostructure grows, new triangular faces are created and can be used to initiate the growth of branches from the main structure via the same process of sequential selfassembly. By design, each face encodes for a specific particle. We have created a new structure with predetermined branching of an unmodified tetrahelix (with $Z=10$ ). The branched structure is encoded by initiating the growth of a new tetrahelix at one of the side faces. Since there is a 10 particle repeat in our design, there is one side branch per every 10 particles in the backbone. Four such repeat units are shown in the Fig. 10 (a). This structure is made with the four seed particles plus 17 other particle types. This kind of branching could be used to make designer gels composed of nanoparticles. In a similar manner, one can create a recursive design where one of the structures acts as a seed for 


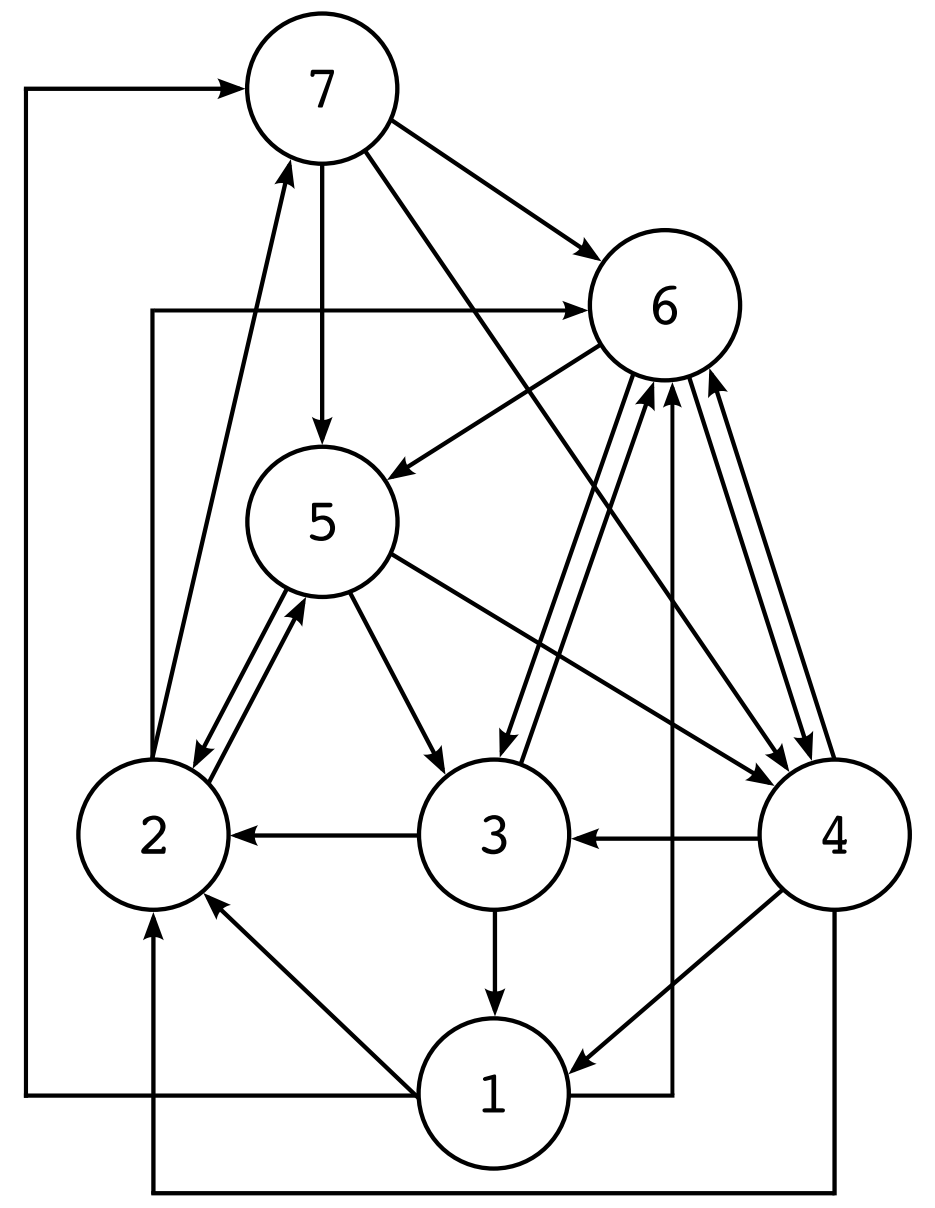

FIG. 9. Connectivity graph showing our design of the modified tetrahelix, structure shown in Fig. 8(c). Unlike the original tetrahelix design in which particle $n$ has a spider that binds to particles $n-1, n-2$ and $n-3$, there is a rewiring of regulations for particles 1,2 and 3 that result in a substantial shape change.

another. For instance Fig. 10 (b), shows a design in which an unmodified tetrahelix made of $\mathrm{Z}=10$ particles is followed by a 7 -particle motif that encodes a $90^{\circ}$ turn, followed by another 10-particle tetrahelix, etc. This process repeats indefinitely producing a staircase pattern shown in the figure.

\section{DISCUSSION}

In summary, we propose a new general strategy for a bottom-up design of an arbitrary mesostructure: a sequential programmable self-assembly. In this approach, the target structure grows one particle at a time, and the position of each particle is predetermined by its $D$ upstream neighbors. We also demonstrated that the simplest realization of this approach, 

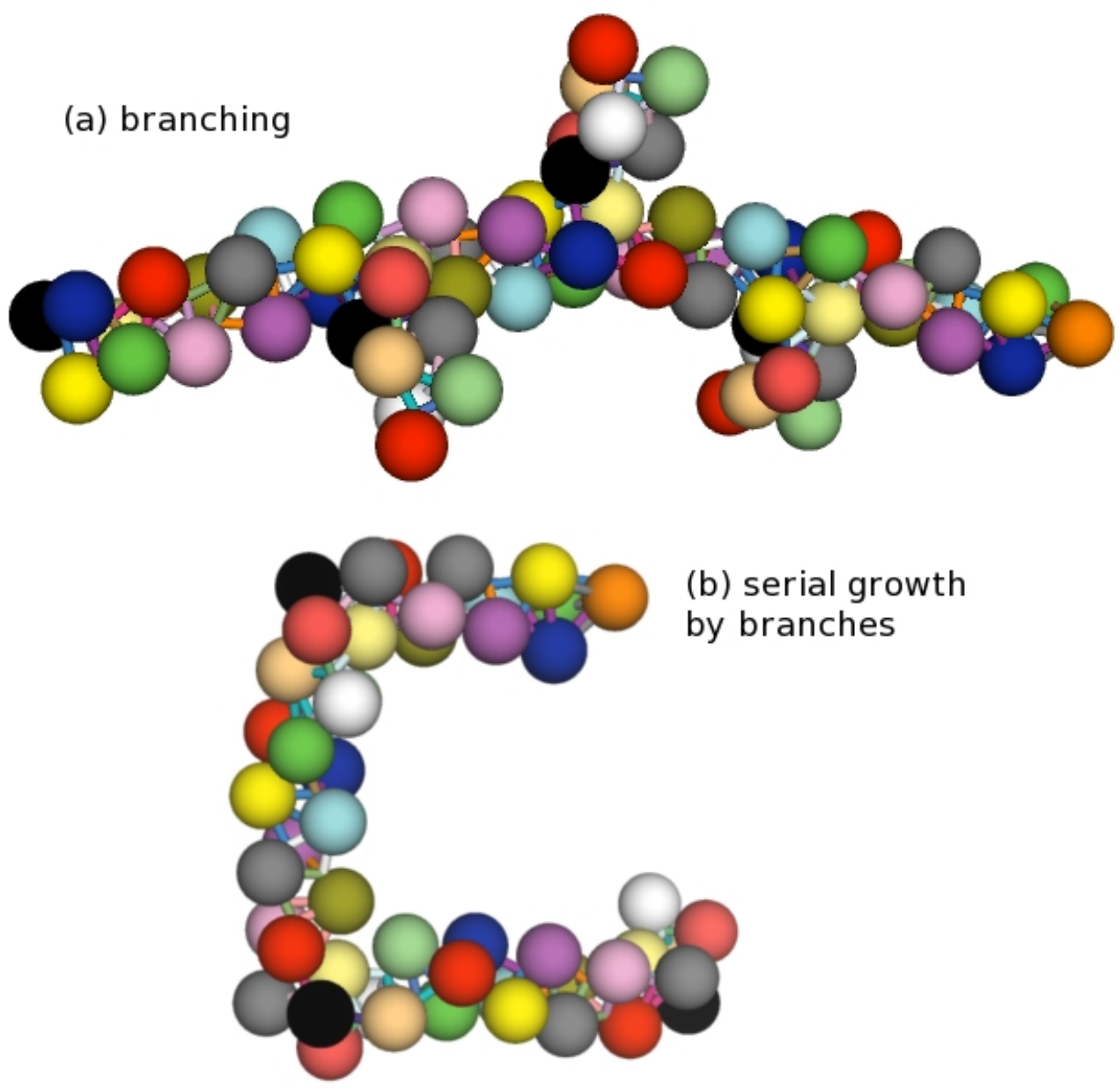

FIG. 10. As the structure forms, new faces are created which can serve as initiators for branches. (a) The white particles bind to the original structure and sequential cooperative growth begins again with a new finite set of particles (7 in total, terminating with red). (b) The recursive design is shown to produce an alteration of a 10-particle tetrahelix with 7 -particle $90^{\circ}$ turn motif.

based on additive interparticle interactions, has intrinsic limitations that leads to a high error rate which makes it impractical, especially in 3D. To tackle this problem, we introduced cooperative interparticle interactions that can greatly suppress the error rate. Cooperative binding is omnipresent in biological systems: they are essential for biochemical regulations and signaling. Here we propose the design of a DNA-based construct (DNA "spider") that possesses built-in binding cooperativity, and therefore can be used to control the sequential assembly.

The design process itself is very similar to playing with a famous GEOMAG construction 
toy (made of magnetic rods and metallic beads). In fact, most GEOMAG designs can in principle be programmed by using spider-functionalized particles, and, according to our results, successfully self-assembled. Certainly, large non-trivial designs would require large collections of constituent particle types. Their number can be reduced if the structure has certain symmetry, as was demonstrated in the case of the tetrahelix and its derivatives.

One has to emphasize that according to our results, successful error-free assembly requires each particle to have no more than one spider element attached to it. Introduction of a small fraction of particles with two and more identical spider sites predictably resulted in local "mutations" of the target structures. Therefore, purification of the original pool of the building blocks is essential. One possibility of achieving this is to start with a highly asymmetric mixture of a small number of free spiders and large number of particles to be functionalized. This will ensure that multi-spider particles are rare. Fortunately, our simulations indicate that adding "spectator" particles that are functionalized isotropically with DNA, but do not have any spider sites, does not lead to any errors in assembly. Still, technically it is relatively easy to separate particles that do have spiders attached to them from those without. After that, the spider functionalization procedure can be repeated again with the latter group. The result of such a cycling would be an efficient "mass production" of spider-functionalized particles with a very small fraction of double-functionalized ones.

DNA spider is certainly not the only possible implementation of cooperative interactions suitable for sequential self-assembly. For instance, a number of recent theoretical and experimental studies discuss various schemes that involve particles with mobile linkers ${ }^{21-24}$. Those linkers may be of arbitrary nature, e.g. DNA-based. One can use that approach as an alternative to our DNA-spider scheme, by introducing three linker types, A,B, C with mutual interactions. Once linkers of two type, e.g. $A$ and $B$, are being used for making the respective interparticle bonds, the third type $C$ becomes unbound and therefore more reactive. This scenario of cooperative binding is most suitable for larger, micron-size colloidal and emulsion systems.

We anticipate that the designed DNA-based cooperative interactions can be utilized beyond controlling the NP and colloidal self-assembly. In particular, they open a prospect of an intelligent functional matter that undergoes transformations in response to the changing environment. Similarly to biochemical networks, one can expect the cooperative binding to be essential for sharp and robust response to such changes. 
Acknowledgments. This work benefited from discussions with O. Gang, P. Chaikin, M. Hybertsen. This research used resources of the Center for Functional Nanomaterials, which is a U.S. DOE Office of Science Facility, at Brookhaven National Laboratory under Contract No. DE-SC0012704.

\section{REFERENCES}

${ }^{1}$ C. Mirkin, R. Letsinger, R. Mucic, and J. Storhoff, Nature 382, 607 (1996).

${ }^{2}$ A. Alivisatos, K. Johnsson, X. Peng, T. Wilson, C. Loweth, M. Bruchez, and P. Schultz, Nature 382, 609 (1996).

${ }^{3}$ S. Y. Park, A. K. R. Lytton-Jean, B. Lee, S. Weigand, G. C. Schatz, and C. A. Mirkin, Nature 451, 553 (2008).

${ }^{4}$ P. Biancaniello, A. Kim, and J. Crocker, Phys. Rev. Lett. 94, 058302 (2005).

${ }^{5}$ D. Nykypanchuk, M. M. Maye, D. van der Lelie, and O. Gang, Nature 451, 549 (2008).

${ }^{6}$ S. Angioletti-Uberti, B. M. Mognetti, and D. Frenkel, Nature Materials 11, 518 (2012).

${ }^{7}$ R. J. Macfarlane, B. Lee, M. R. Jones, N. Harris, G. C. Schatz, and C. A. Mirkin, Science 334, 204 (2011).

${ }^{8}$ A. V. Tkachenko, Phys. Rev. Lett. 89, 148303 (2002).

${ }^{9}$ F. J. Martinez-Veracoechea, B. M. Mladek, A. V. Tkachenko, and D. Frenkel, Phys. Rev. Lett. 107, 045902 (2011).

${ }^{10}$ N. A. Licata and A. V. Tkachenko, Europhys. Lett. 84, 20010 (2008).

${ }^{11}$ G. Meng, N. Arkus, M. P. Brenner, and V. N. Manoharan, Science 327, 560 (2010).

${ }^{12}$ S. Hormoz and M. P. Brenner, Proc. Natl. Acad. Sci. USA 108, 5193 (2011).

${ }^{13}$ A. V. Tkachenko, Phys. Rev. Lett. 106, 255501 (2011).

${ }^{14}$ A. Murugan, J. Zou, and M. P. Brenner, Nature Communications 6, 6203 (2015).

${ }^{15}$ W. M. Jacobs, A. Reinhardt, and D. Frenkel, Proceedings of the National Academy of Sciences 112, 6313 (2015).

${ }^{16}$ J. D. Halverson and A. V. Tkachenko, Phys. Rev. E 87, 062310 (2013).

${ }^{17}$ M. E. Leunissen, R. Dreyfus, F. C. Cheong, G. D. G., R. Sha, N. C. Seeman, and P. M. Chaikin, Nature Materials 8, 595 (2009/07).

${ }^{18}$ P. Rothemund, Nature 440, 297 (2006).

${ }^{19}$ A. Boerdijk, Philips Res. Rep. 7, 30 (1952). 
${ }^{20}$ H. Coxeter, Regular Complex Polytopes (Cambridge University, 1974).

${ }^{21}$ P. A. Beales and T. K. Vanderlick*, The Journal of Physical Chemistry A 111, 12372 (2007).

${ }^{22}$ S. Angioletti-Uberti, P. Varilly, B. M. Mognetti, and D. Frenkel, Phys. Rev. Lett. 113, $128303(2014)$.

${ }^{23}$ S. A. J. van der Meulen and M. E. Leunissen, Journal of the American Chemical Society 135, $15129(2013)$.

${ }^{24}$ L. Parolini, B. M. Mognetti, J. Kotar, E. Eiser, P. Cicuta, and L. Di Michele, Nat. Commun 6, 5948 (2015). 